\title{
Theranostics
}

2016; 6(10): 1601-1602. doi: 10.7150/thno.16337

Editorial

\section{The Pursuit of Theranostics with CEST MRI}

\author{
Mark Marty Pagel ${ }^{凶}$ \\ Contrast Agent Molecular Engineering Laboratory, Department of Medical Imaging University of Arizona \\ $\bowtie$ Corresponding author: Tel: (520)-404-7049. Email: mpagel@u.arizona.edu \\ (C) Ivyspring International Publisher. Reproduction is permitted for personal, noncommercial use, provided that the article is in whole, unmodified, and properly cited. See \\ http://ivyspring.com/terms for terms and conditions.
}

Received: 2016.05.31; Accepted: 2016.05.31; Published: 2016.06.18

Molecular imaging has often provided tantalizing potential for tracking theranostic agents during in vivo studies. Each in vivo imaging modality has its limitations, which has impeded the application of molecular imaging towards theranostics research. For example, Magnetic Resonance Spectroscopy (MRS) has been used to detect drug delivery with great molecular specificity due to the unique MR frequencies for each agent. ${ }^{1}$ However, MRS suffers from poor detection sensitivity, often requiring toxic concentrations of the agent. Magnetic Resonance Imaging (MRI) overcomes poor detection sensitivity by directly detecting water that is abundant in soft tissues. Theranostic agents can be labeled or packaged with metals that can influence the water signal detected with MRI. ${ }^{2}$ Unfortunately, many conditions affect MRI signals, so the specificity of detecting a metal-labeled agent is often lost when detecting the influence of the agent on the MRI signal of the water.

Chemical Exchange Saturation Transfer (CEST) MRI is a hybrid of MRS and MRI that has evolved from the chemistry benchtop, to small animal imaging studies, to clinical radiology studies during the past century. ${ }^{3}$ Saturation at the specific MR frequency of a proton (a hydrogen atom) on a theranostic agent causes the proton's coherent magnetization to disappear. This disappearance in MRI signal is then transferred to water after the agent's proton undergoes chemical exchange with a water molecule. The resulting decrease in water signal can be detected with good sensitivity using standard MRI methods. Despite substantial interest in CEST MRI, concerns have been raised that the intermediate detection sensitivity and specificity may only result in inadequate sensitivity and specificity, resulting in very limited utility of this MRI method for non-invasively tracking theranostic agents during in vivo studies.

A recent study by Prof. Guanshu Liu and colleagues at the Johns Hopkins University School of Medicine has shown promise that CEST MRI can successfully track a theranostic agent with adequate sensitivity and excellent specificity. ${ }^{4}$ Citicoline (cytidine-5'-diphosphocholine) is a neurodegenerative therapeutic agent that has shown significant neuroprotective effects afer ischemic stroke. Varying results have been attributed to variable delivery of citicoline, and various routes of administration have been proposed to improve delivery. Thus, there is a justifiable need to track the delivery of citicoline as a theranostic agent. Ideally, this agent should be tracked without labeling the molecule with an imaging probe, which inevitably raises concerns about altered efficacy and/or pharmacokinetics of the theranostic agent.

Fortunately, citicoline contains cytosine that has previously been shown to act as a CEST agent. The amine group and two hydroxyl groups of citicoline have labile protons that readily exchange with water protons. Liu and colleagues showed that selective saturation at the MR frequencies of these specific protons, followed by the detection of decreased water signal, resulted in the sensitive detection of CEST signals from citicoline. Importantly, this CEST detection arises from the labile protons of the agent, obviating the need for labeling the molecule with an imaging probe. Therefore, this drug acts as a CEST agent, and thereby qualifies as a theranostic agent.

As a major breakthrough in CEST MRI reseach, the investigators were able to show sufficiently sensitive detection of citicoline during CEST MRI studies with a rat model of transient focal cerebral ischemia. Initial studies in chemical solution showed that less than $1 \mathrm{mM}$ of citicoline could be reliably 
detected. To offset concerns that in vivo imaging would require stronger CEST signals than ideal conditions in chemical solutions, Liu and colleagues packaged citicoline in a liposome, concentrating the agent 500,000-fold within the liposome's volume. Interarterial injection of this nanoscale, theranostic agent resulted in good CEST MRI detection of an estimated $2 \mathrm{mM}$ of citicoline within ischemic brain tissues. To further enhance delivery, the investigators also tested a citicholine-containing liposome that was conjugated to antibodies that target vascular cell adhesion molecule 1 (VCAM-1), which is overexpressed on the endothelial layer of inflamed vessels during cerebral ischemia. Intravenous delivery of the targeting formulation also demonstrated detection of citicoline with CEST MRI just 24 hours after initiating the stroke in the rat model.

Several challenges remain before CEST MRI can be routinely applied for clinical studies of theranostic agents. In particular, the quantification of CEST signals is a complicated exercise, because CEST is affected by the concentration of water and MR properties of the tissue, as well as experimental conditions such as the amplitude and homogeneity of the saturation power applied to generate the CEST effect. Liu and colleagues have mitigated some complications in their experimental design, such as measuring pre- and post-injection CEST signals to differentiate endogenous CEST effects from CEST signals of the exogenous theranostic agent. Furthermore, the investigators of this study propose to use innovative CEST detection methods that can be 'tuned' for preferential detection of agents with specific chemical exchange rates, which may improve the quantification and specificity of detecting theranostic agents in vivo.

Despite this outstanding in vivo imaging demonstration with citicoline, this CEST MRI technique may be challenging to apply to the study of other theranostic agents. As a minor concern, Liu and colleagues used a liposomal formulation during in vivo studies, which provided a large delivery of drug payload to the ischemic tissue. A large payload of agent appears to be a requirement to achieve sufficient CEST detection, which may limit this technique to nanoscale theranostic agents. This is only a minor consideration, because drug nanocarriers are commonly employed in theranostics research so that CEST MRI may still have great utility for tracking these drug formulations. As another concern, citicoline is a well-studied neuroprotective agent with an established safety profile at a very high clinical dose of 500-200 mg daily intravenous administration. This condition suggests that CEST MRI may be limited to tracking theranostic agents that have tolerability at very high doses. As Liu and colleagues indicate in their study, these challenges justify the need for additional studies, including eventual clinical studies.

Molecular imaging research often requires a creative approach that is highly multi-disciplinary. This CEST MRI study by Liu and colleagues certainly exemplifies these characteristics, because this this creative team of researchers combined chemistry, pharmaceutical sciences, biomedical imaging, and neurobiology to achieve their successful studies. Moreover, proposing to detect a drug with CEST MRI would seem audacious due to the great difference in drug concentrations vs. CEST agent concentrations. Yet this high-risk approach has certainly resulted in a high-reward result of providing a new molecular imaging modality for developing theranostics. For these reasons, Prof. Guanshu Liu and his research collaborators should be commended for their pursuit of theranostics with CEST MRI.

\section{References}

1. Lu ZR. In vivo imaging of dosage forms. Drugs Pharma Sciences 2009, 188:181-201.

2. Gobbo OL, Sjaastad K, Radomski MW, Volkov Y, Prina-Mello A. Magnetic nanoparticles in cancer theranostics. Theranostics 2015, 5:1249-1263.

3. Liu G, Song X, Chan KWY, McMahon MT. Nuts and bolts of chemical exchange saturation transfer MRI. NMR Biomed 2013, 26:810-828.

4. Liu H, Jablonska A, Li Y, Cao S, Liu D, Chen H, Van Zijl PC, Bulte JWM, Janowski M, Walczak P, Liu G. Label-free CEST MRI Detection of Citicoline-Liposome Drug Delivery in Ischemic Stroke. Theranostics 2016; 6(10):1588-1600. 